\title{
Effect of Mn doping on structural and dielectric properties of $\mathrm{SmFeO}_{3}$
}

\author{
Shahid Husain*, Ali O.A. Keelani and Samiya Manzoor \\ Department of Physics, Aligarh Muslim University, Aligarh-202002 INDIA \\ *Email: s.husain@lycos.com
}

In recent past transition metal oxides have attracted a great deal of attention for their various properties, such as ferromagnetism, ferroelectricity and superconductivity. Among these oxides, the rare-earth orthoferrites, $\mathrm{ReFeO}_{3} \quad(\mathrm{Re}=$ rare-earth), with distorted perovskite structures are of great scientific importance because these materials not only exhibit fascinating magnetic properties but also offer a wide range of new applications for next generation storage devices. Among all the rare earth orthoferrites, $\mathrm{SmFeO}_{3}$ possesses the spin reorientation phase transition high above room temperature and $\mathrm{Sm} \mathrm{3p}$ magnetic moments orders at relatively higher temperature. Nanocrystalline samples of $\mathrm{SmFe}_{1-\mathrm{x}} \mathrm{Mn}_{\mathrm{x}} \mathrm{O}_{3} \quad(x=0.0$, $0.1,0.2$ and 0.30 ) were synthesized by solid state reaction route to study their structural and dielectric properties. X-ray diffraction (XRD) patterns are recorded to confirm phase purity. These samples are found to be orthorhombic with Pbnm space group. The lattice parameter a, $\mathrm{b}$ and $\mathrm{c}$ are determined using Rietveld refinement. These parameters and unit cell volume decrease slightly with the increase in $\mathrm{Mn}$ concentration. The strain and crystallite size, calculated using Williamson-Hall-plots, exhibit an increase with the increase in $\mathrm{Mn}$ concentration. Dielectric responses of our samples have been studied in the light of universal dielectric response (UDR) model [1]. The value of real part of dielectric constant $\left(\varepsilon^{\prime}\right)$ is found to be enhanced with Mn doping. In order to verify the UDR behavior, the log (frequency) versus $\log$ (frequency $\mathrm{x} \varepsilon^{\prime}$ ) graphs have been plotted (Figure 1). We have found the best linear fit for the host $\left(\mathrm{SmFeO}_{3}\right)$ sample in the whole frequency range and for the other samples the linear behavior is observed at low frequency regions only.

Lattice parameters are determined using PowderX software using Rietveld refinement are found to be $\mathrm{a}=5.3999 \AA, \mathrm{b}=5.5988 \AA$ and $\mathrm{c}=7.7153 \AA$ [2]. W-H analysis of our samples assuming
UDM, reveals that the strain and size of particle increase with the increase in Mn concentration (Figure 2).
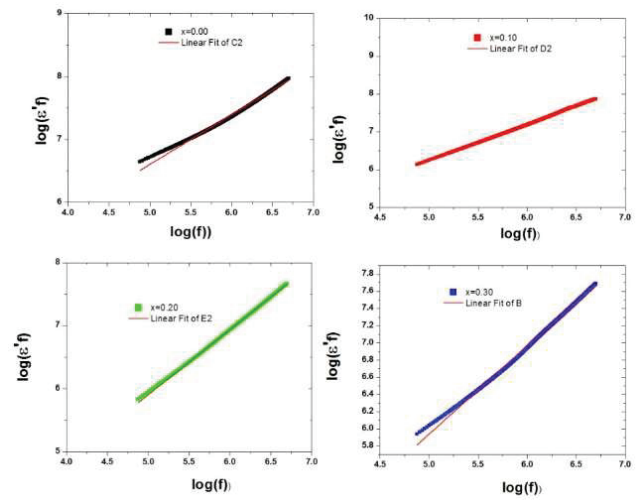

Figure 1: Plots of $\log (f)$ versus $\log \left(\varepsilon^{\prime} \times f\right)$ of different samples $\mathrm{SmFe}_{1-\mathrm{x}} \mathrm{Mn}_{\mathrm{x}} \mathrm{O}_{3}(\mathrm{x}=0.0,0.10,0.20 \&$ $0.03)$
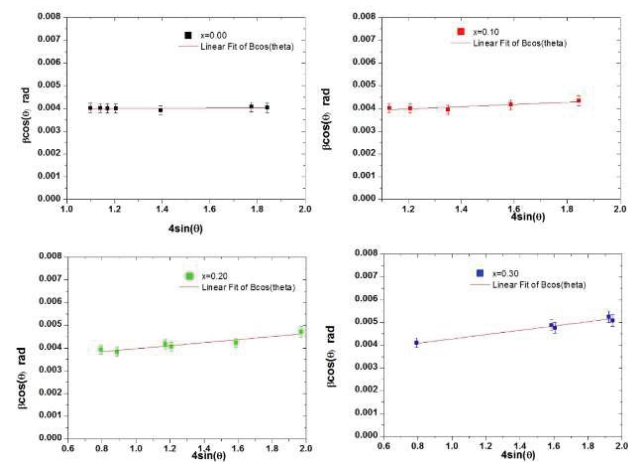

Figure 2: The $\mathrm{W}-\mathrm{H}$ analysis of $\mathrm{SmFe}_{1-\mathrm{x}} \mathrm{Mn}_{\mathrm{x}} \mathrm{O}_{3}(\mathrm{x}=0.0$, $0.1,0.2 \& 0.3$ ) samples with assuming UDM (solid lines: Fit to the data). The strain is extracted from the slope and the crystalline size is extracted from the yintercept of the fit

\section{References}

1. A.K. Jonscher, J. Phys. D: Appl. Phys. 32 (1999) R57-R70.

2. M. Siemons and U. Simon. Solid State Phenomena Vol. 128 (2007) pp 225-236. 2007. 